\title{
Characterization of Rice Genotypes Based on Physical, Chemical and Molecular Methods
}

\author{
E. Sudeep Kumar ${ }^{1 *}$, S. N. Vasudevan ${ }^{2}$, N. M. Shakuntala ${ }^{1}$, S. R. Doddagoudar ${ }^{1}$, \\ B. G. MasthanReddy ${ }^{3}$ and K. Mahantashivayogayya ${ }^{3}$ \\ ${ }^{1}$ Department of Seed Science \& Techonology, ${ }^{3}$ ARS Gangavath, UAS Raichur, India \\ ${ }^{2}$ Director of Research, ZARS Mandya, UAS Bengaluru, India \\ *Corresponding author
}

Keywords

Biovis seed image analyzer, Phenol test, $\mathrm{FeSO}_{4}$ test, $\mathrm{KOH}$ test, $\mathrm{NaOH}$ test, Peroxidase test, SSR markers

Article Info

Accepted:

12 December 2020

Available Online:

10 January 2021

\section{A B S T R A C T}

The present investigation was carried out to know the response and characterization of rice genotypes by physical, chemical and molecular methods. Seeds of 17 rice genotypes were analysed for seed dimension using Biovis seed image analyzer. The mean performance for seed length varied from $9.28 \mathrm{~mm}$ in genotype RYC-230 to $11.50 \mathrm{~mm}$ in genotypeMT-4420 and seed width varied from $2.64 \mathrm{~mm}$ in genotype IET-18299 to $4.85 \mathrm{~mm}$ in genotype MT-4420. The mean seed length/seed width ratio ranged from 2-37 to 3-53 among the genotypes. Rice genotypes also well responded to chemical tests such as phenol test, $\mathrm{FeSO}_{4}$ test, $\mathrm{NaOH}$ test and $\mathrm{KOH}$ test and helpful in grouping of the rice genotypes. Of the 22 single sequence repeats (SSR) markers used in the study, all markers were able to distinguish rice genotypes and may be utilized to develop new varieties during crop improvement programme.

\section{Introduction}

Rice (Oryza sativa L.) is the primary cereal crop grown in many countries especially in Asia. The increasing population rate challenges the world's food requirements and an area expansion under rice likely to decline nowadays because of urbanization, climate change and high-value agriculture. Hence, more stress has been given for rice crop improvement since ages, which has resulted in the release of a large number of varieties with improved yield or tolerance to biotic and abiotic stresses. As India is a signatory for the WTO, and The Protection of Plant Varieties and Farmer's Rights (PPV \& FR) Act, 2001 came into existence, varietal characterization and identification became more significant in the present scenario because India is having rich gene pool. Many countries including International Seed Testing Association (ISTA), Switzerland have been conducting elaborate research for developing standard chemical tests, physiological tests and use of 
various biomolecular markers for varietal identification in crops.

The shape, size and colour of seeds are normally employed to identify rice varieties. Use of computer-based image analysis is a good alternative to visual identification. Hiremath (2013) grouped rice genotypes based on seed length and seed width using Marvin seed image analyser. Similarly, Maruthi (2016) classified 180 paddy genotypes based on seed length and seed width using Biovis seed image analyser. Various studies on the characterization of cultivars confirmed that the response of seed and seedlings to various chemical tests viz., phenol test, modified phenol test, ferrous sulphate test, potassium hydroxide test offer a wider variability and can be used in the characterization of genotypes.

The simple, reliable and quick chemical tests can be used for varietal identification in rice crop (Vijayalakshmi and Vijay, 2009).The chemical tests gave the stable results and could be effectively used for cultivar differentiation and determining the varietal purity of rice for routine testing in seed testing laboratories as some of the cultivars showed a distinct response to these phenol test (Anitalakshmi et al., 2014). Masuthi et al., (2015) used 32 paddy genotypes for phenol test, out of which 12 genotypes showed no colour change, 15 genotypes were light brown, 8 genotypes were brown and 6 genotypes were dark brown in colour. Saharan (1991) classified 33 genotypes of rice into four groups viz., brown spot, brown streaks, grey spot and grey streaks kernels by using 1.5 per cent ferrous sulphate solution. Similar classification was also done by Hiremath (2013) in rice genotypes and Raju et al., (2017) in rice hybrid with $\mathrm{FeSO}_{4}$ test. Among the ten rice genotypes, four genotypes (Gangavatimallige, Gangavatisanna, Gidda emergency and JGL-1798) showed light brown and six genotypes (Mysore mallige, GGV-05-01, Gangavati emergency, CSR-22, BPT-5204 and Ratansagar) showed yellow colour reaction with $\mathrm{KOH}$ test (Hiremath, 2013). The similar grouping was done by Masuthi et al., (2015) in rice genotypes and Raju et al., (2017) in rice hybrids by using $\mathrm{KOH}$ test.

Therefore, an investigation was carried with the seventeen rice genotypes to characterize and to know the response of rice genotypes to various physical, physiological, chemical tests and by molecular markers, which helps in the determination of cultivar purity in rice.

\section{Materials and Methods}

The present investigation was carried out during the year 2016-17 in the Department of Seed Science and Technology, College of Agriculture, Raichur. Seventeen rice genotypes $\left(\mathrm{G}_{1}: \mathrm{RYC}-230, \mathrm{G}_{2}: \mathrm{GNV}-1405\right.$, $\mathrm{G}_{3}$ :GNV-1089, G 4 :GNV-1301, G5:GNV-0501, G6:GNV-1109, G G $_{7}$ :MT-4253, G 22066, G9:MT-4021, G 10 :BPT-5204, G 11 :IET18299, G $\mathrm{G}_{12}$ :MT-4420, $\mathrm{G}_{13}$ :MTU-1010, $\mathrm{G}_{14}$ :MT-4541, G $\mathrm{G}_{15}$ :IET-19251, G 16 :MAS-26 and $\mathrm{G}_{17}$ :MAS-946-1)were used for image analysis, chemical tests and molecular studies.

\section{Physical method by Biovis seed image analyzer}

The seed image analyzer provides more information than a traditional counting device. It works fast, noiseless and easily operated. The features of varieties may be stored in a pre-configured, able to learn database for recognizing and identifying main seeds, foreign seeds and non-seed particles. The Biovis seed image analyzer is a device that comprises a scanner, which scans the images and captures the same with their length, width, area, perimeter and roundness with coloured images. The special Biovis 
image analysis software offers a lot of possibilities, to adjust the recording of the analysis results and statistical interpretation according to the requirements and demands of the user. Thus, it is suitable for the analysis of the seeds of many crops.

\section{Seed length}

100 seeds of each genotype were measured using Biovis image analyzer. The mean value of seed length was expressed in millimetre and was grouped according to DUS test guidelines for rice as very short $(<6.0 \mathrm{~mm})$, short $(6.1-8.5 \mathrm{~mm})$, medium $(8.6-10.5 \mathrm{~mm})$, long (10.6-12.5 $\mathrm{mm})$ and very long $(>12.5$ $\mathrm{mm})$

\section{Seed width}

100 seeds of each genotype were measured using Biovis image analyzer. The mean value of seed length was expressed in millimetre and was grouped according to DUS test guidelines for rice as very narrow $(<2.0 \mathrm{~mm})$, narrow (2.1-2.5 mm), medium (2.6-3.0 mm), broad (3.1-3.5 $\mathrm{mm})$ and very broad (> 3.5 $\mathrm{mm})$.

\section{By chemical tests}

The chemical tests find variations among the seeds and seedlings of different crop varieties. These tests do not require technical expertise or training and can be completed in a relatively short period time. The results of these tests are usually distinct, easily interpreted and help in the grouping of the genotypes.

\section{Phenol test}

The standard phenol test for varietal purity testing suggested by Walls (1965) was followed. Four replicates of 50 seeds each were soaked in distilled water for $18 \mathrm{~h}$. The seeds were then placed in Petri dishes containing filter paper moistened with $4 \mathrm{ml}$ of 2 per cent phenol solution and kept at room temperature $\left(28^{\circ} \mathrm{C}\right)$. After $24 \mathrm{~h}$, seeds were examined and grouped into different colour classes viz., as no colour change, light brown, brown and dark brown.

\section{Ferrous Sulphate $\left(\mathrm{FeSO}_{4}\right)$ test}

As described by Gupta and Agrawal (1988), fifty seeds each in four replications were soaked in 1.5 per cent FeSO4 solution for $4 \mathrm{~h}$ under ambient condition and later the seeds were taken out and the excess moisture was removed using blotting paper before evaluation. The seeds were examined for colour reaction and the distinct colour groups were recorded as brown spots, grey spots and grey streaks.

\section{Potassium hydroxide (KOH) test}

Seeds were soaked in 5 per cent $\mathrm{KOH}$ solution in 4 replications of 50 seeds each and kept at room temperature $\left(28^{\circ} \mathrm{C}\right)$ for three hours. The colour change of the $\mathrm{KOH}$ solution was observed and based on the colour reaction the genotypes were divided into two groups viz., deep wine red and no staining.

\section{Sodium hydroxide $(\mathrm{NaOH})$ test}

Four replications of 50 seeds each were soaked in 5 per cent $\mathrm{NaOH}$ solution for one hour and the change in colour of the solution was observed. Based on the colour developed, varieties were classified into three groups viz., dark yellow, light yellow and no colour.

\section{Peroxidase $\left(\mathrm{H}_{2} \mathrm{O}_{2}\right)$ test}

It was studied as per the procedure given by Buttery and Buzzell (1968). Ten seed coats were removed and placed separately in the 
test tube, with three replications for genotypes and added 10 drops of 0.5 per cent Guaiacol solution into the test tube, after ten minutes one drop of 0.1 per cent solution of hydrogen peroxide $\left(\mathrm{H}_{2} \mathrm{O}_{2}\right)$ was added and the reactions were noted exactly after sixty seconds. The colouration due to peroxidase activity was observed to group the varieties as present and absent of brown colour solution.

\section{By Molecular markers}

Twenty two Rice Microsatellite (RM) markers were used to study the polymorphism among the seventeen rice genotypes (Table 1.) that are screened under Direct seeded rice (DSR) method. The method followed for DNA extraction was Modified Cetyltrimethyl Ammonium Bromide (CTAB) method (Cao and Oard, 1997).

\section{Results and Discussion}

Grouping of genotypes based on seed morphology using Biovis Seed Image Analyzer

\section{Seed length $(\mathrm{mm})$}

Seeds of 17 rice genotypes were analyzed for seed dimension using Biovis seed image analyser. The mean performance for seed length varied from $9.28 \mathrm{~mm}$ in RYC-230 to $11.50 \mathrm{~mm}$ in MT- 4420 and seed width varied from $2.64 \mathrm{~mm}$ in IET-18299 to $4.85 \mathrm{~mm}$ in MT-4420 (Table 2.\& 3.). The genotypes viz., RYC-230, GNV-1405, GNV-1089, GNV-0501, GNV-1109, BPT-5204, IET-18299 and IET-19251 are having medium seed length and genotypes GNV-1301, MT-4253, IET22066, MT-4021, MT-4420, MTU-1010, MT4541, MAS-26 and MAS-946-1 possess longer seed length and none of them are very short, short and very long. Similarly, seed width in rice genotypes viz., RYC-230, GNV1405, GNV-05-01, BPT-5204, IET-18299 and
IET-19251 was medium while, genotype GNV-1089 was broad type and genotypes GNV-1301, GNV-1109, MT-4253, IET22066, MT-4021, MT-4420, MTU-1010, MT4541, MAS-26 and MAS-946-1 falls under very broad width category. None of the genotypes in seed width was a very narrow and narrow group. So also, the mean seed length/seed width ratio ranged from 2.37 to 3.53among the genotypes. Four genotypes were grouped as semi-spherical, five genotypes as semi-long and remaining eight genotypes were grouped as elongated. A similar study of image analysis on seed length and width were carried out by Paulsen et al (1989) in maize, Zayas et al., (1989, 1996), Utku et al., (1998) in rice, Suchowilska and Wiwari (2006) and Sainis et al., (2009) in wheat. Variations based on seed size and shape and other seed parameters have also been reported by Anuradha et al., (2009), Iwata et al., (2010), Hiremath (2013) and Maruthi (2016) in paddy.

\section{Identification of rice genotypes through chemical tests}

\section{Phenol test}

Based on seed colouration with phenol, the rice genotypes studied were grouped into three categories viz., no colour, light brown, brown and dark brown. Three genotypes viz., $\mathrm{G}_{2}$ :GNV-1405, $\mathrm{G}_{11}$ :IET-18299 and $\mathrm{G}_{14}$ :MT4541 showed no colour, four genotypes $\left(\mathrm{G}_{1}: \mathrm{RYC}-230, \mathrm{G}_{4}: \mathrm{GNV}-1301, \mathrm{G}_{6}: \mathrm{GNV}-1109\right.$ and $\mathrm{G}_{9}: \mathrm{MT}-4021$ ) showed brown colour, two genotypes (G3-GNV- 1089 and G8-IET22066) showed dark brown and eight genotypes $\left(\mathrm{G}_{5}: \mathrm{GNV}-05-01, \mathrm{G}_{7}\right.$ :MT-4253, $\mathrm{G}_{10}$ :BPT-5204, $\mathrm{G}_{12}$ :MT-4420, $\mathrm{G}_{13}$ :MTU1010, G $\mathrm{G}_{15}$ :IET-19251, G $\mathrm{G}_{16}$ :MAS-26 and $\mathrm{G}_{17}$ :MAS-946-1) showed light brown colour (Fig.1). Similarly, the genotypes were grouped earlier based on phenol colour reaction in rice by Meshram and Rahangdale 
(1988), Wang and Shen (1992), Vanagamudi et al., (1988), Jaiswal and Agarwal (1995), Bora et al., (2008), Devi Singh et al., (2011), Tiwari et al., (2013) and Masuthi et al., 2015) in rice. The present findings reveal that phenol test could be used as a simple, quick and cheap method for grouping paddy genotypes. The difference in the phenol colour reaction of hulls seems to be due to the differences in the genetic background, presumably concerning the enzyme system. Seed colouration with phenol is one of the important qualitative characteristics which is not affected by the environmental condition.
The result of phenol test is usually distinct and easily interpreted. Walls (1965) reported that the phenol colour reaction depends on the quality and quantity of oxidase enzymes present in seeds. Whereas, Takahashi and Hamza (1983) reported monophenol oxidase was extremely localized in grains even though it is present in all other plant parts. Phenol colour reaction, which is an index of polyphenol oxidase activity, has been utilized to distinguish the crop varieties by earlier workers Joshi and Banerjee (1970) in wheat, Abrol and Uprety (1972) and Chauhan and Nanda (1984) in rice.

Table.1 List of SSR primers used to study the polymorphism among rice genotypes

\begin{tabular}{|c|c|c|c|}
\hline SI.No. & Marker & Forward primers (5'-3') & Reverse primers ( $\left(3^{\prime}-5^{\prime}\right)$ \\
\hline 1. & RM241 & Gagccaaataagatcgctga & Tgcaagcagcagatttagtg \\
\hline 2. & RM257 & Cagttccgagcaagagtactc & Ggatcggacgtggcatatg \\
\hline 3. & RM224 & Atcgatcgatcttcacgagg & Tgctataaaaggcattcggg \\
\hline 4. & RM219 & Cgtcggatgatgtaaagcet & Catatcggcattcgectg \\
\hline 5. & RM252 & Ttcgctgacgtgataggttg & Atgacttgatcccgagaacg \\
\hline 6. & RM562 & Cacaacccacaaacagcaag & Cttcccccaaagttttagcc \\
\hline 7. & RM140 & Tgcctcttcectggetccectg & Ggcatgccgaatgaaatgcatg \\
\hline 8. & RM448 & Tctgatcttgatgcaggcac & Tctcccgatttggacagatc \\
\hline 9. & RM578 & Ggcgtcgtgttttctctctc & Caaaaaggaggagcagatcg \\
\hline 10. & RM286 & Ggcttcatctttggcgac & Ceggattcacgagataaactc \\
\hline 11. & OSR28 & Agcagctatagcttagctgg & Actgcacatgagcagagaca \\
\hline 12. & RM311 & Tggtagtataggtactaaacat & Tcctatacacatacaaacatac \\
\hline 13. & RM171 & Aacgcgaggacacgtacttac & Acgagatacgtacgcctttg \\
\hline 14. & RM452 & Ctgatcgagagcgttaaggg & Gggatcaaaccacgtttctg \\
\hline 15. & RM13 & Tccaacatggcaagagagag & Ggtggcattcgattccag \\
\hline 16. & RM306 & Caaggtcaagaatgcaatgg & Gccactttaatcattgcatc \\
\hline 17. & RM5389 & Tcttgcatgagagccaacac & Gctattgcgegagattatcc \\
\hline 18. & RM161 & Tgcagatgagaagcggcgcctc & Tgtgtcatcagacggcgctccg \\
\hline 19. & RM44 & Acgggcaatccgaacaacc & Tcgggaaaacctaccctacc \\
\hline 20. & RM547 & Taggttggcagaccttttcg & Gtcaagatcatcctcgtagcg \\
\hline 21. & RM55 & Cegtcgecgtagtagagaag & Tcccggttattttaaggcg \\
\hline 22. & RM231 & Ccagattatttcetgaggtc & Cacttgcatagttctgcattg \\
\hline
\end{tabular}


Table.2 Seed length, seed width and seed length/ width ratio as influenced by rice genotypes

\begin{tabular}{|c|c|c|c|c|c|c|c|c|c|}
\hline \multirow[t]{2}{*}{ Genotypes } & \multicolumn{3}{|c|}{ Seed length (mm) } & \multicolumn{3}{|c|}{ Seed width (mm) } & \multicolumn{3}{|c|}{ Seed length/ width ratio } \\
\hline & 2015 & 2016 & $\begin{array}{c}\text { Pooled } \\
\text { mean }\end{array}$ & 2015 & 2016 & $\begin{array}{c}\text { Pooled } \\
\text { mean }\end{array}$ & 2015 & 2016 & $\begin{array}{l}\text { Pooled } \\
\text { mean }\end{array}$ \\
\hline $\mathrm{G}_{1}:$ RYC-230 & 9.23 & 9.33 & 9.28 & 2.73 & 2.64 & 2.69 & 3.45 & 3.54 & 3.49 \\
\hline$G_{2}:$ GNV-1405 & 9.40 & 9.53 & 9.47 & 2.90 & 2.80 & 2.85 & 3.25 & 3.40 & 3.32 \\
\hline$G_{3}:$ GNV-1089 & 9.97 & 10.10 & 10.03 & 3.47 & 3.35 & 3.41 & 2.88 & 3.01 & 2.95 \\
\hline$G_{4}:$ GNV-1301 & 10.16 & 10.33 & 10.25 & 3.66 & 3.54 & 3.60 & 2.78 & 2.92 & 2.85 \\
\hline $\begin{array}{l}\text { G }_{5}: \text { GNV-05- } \\
01\end{array}$ & 9.43 & 9.57 & 9.50 & 2.93 & 2.84 & 2.88 & 3.23 & 3.38 & 3.30 \\
\hline G6$_{6}:$ GNV-1109 & 10.43 & 10.57 & 10.50 & 3.93 & 3.80 & 3.87 & 2.65 & 2.78 & 2.72 \\
\hline$G_{7}:$ MT-4253 & 11.33 & 11.47 & 11.40 & 4.83 & 4.67 & 4.75 & 2.35 & 2.46 & 2.40 \\
\hline G8: $_{8}$ IET-22066 & 10.23 & 10.37 & 10.30 & 3.73 & 3.61 & 3.67 & 2.76 & 2.88 & 2.82 \\
\hline G9: $_{9}$ MT-4021 & 11.37 & 11.40 & 11.38 & 4.87 & 4.70 & 4.79 & 2.34 & 2.43 & 2.38 \\
\hline $\mathbf{G}_{10}:$ BPT-5204 & 9.47 & 9.60 & 9.53 & 2.97 & 2.87 & 2.92 & 3.19 & 3.35 & 3.27 \\
\hline $\begin{array}{l}\text { G }_{11}: \\
18299\end{array}$ & 9.18 & 9.32 & 9.25 & 2.68 & 2.59 & 2.64 & 3.47 & 3.59 & 3.53 \\
\hline $\mathrm{G}_{12}: \mathrm{MT}-4420$ & 11.43 & 11.57 & 11.50 & 4.93 & 4.77 & 4.85 & 2.32 & 2.43 & 2.37 \\
\hline $\begin{array}{l}\text { G }_{13}: \\
1010\end{array}$ & 11.34 & 11.34 & 11.34 & 4.83 & 4.67 & 4.75 & 2.35 & 2.43 & 2.39 \\
\hline $\mathrm{G}_{14}: \mathrm{MT}-4541$ & 10.47 & 10.60 & 10.53 & 3.97 & 3.83 & 3.90 & 2.64 & 2.77 & 2.70 \\
\hline $\begin{array}{l}\text { G }_{15}: \text { IET- } \\
19251\end{array}$ & 9.47 & 9.60 & 9.53 & 2.97 & 2.87 & 2.92 & 3.19 & 3.35 & 3.27 \\
\hline G $_{16}:$ MAS-26 & 10.45 & 10.87 & 10.66 & 3.93 & 3.80 & 3.87 & 2.69 & 2.86 & 2.77 \\
\hline $\begin{array}{l}\text { G}_{17}: \text { MAS-946- } \\
1\end{array}$ & 10.33 & 10.47 & 10.40 & 3.83 & 3.71 & 3.77 & 2.78 & 2.82 & 2.80 \\
\hline Mean & 10.22 & 10.35 & 10.29 & 3.72 & 3.59 & 3.65 & 2.84 & 2.96 & 2.90 \\
\hline S.Em \pm & 0.23 & 0.24 & 0.22 & 0.23 & 0.07 & 0.12 & 0.14 & 0.07 & 0.04 \\
\hline CD@1\% & 0.89 & 0.91 & 0.84 & 0.89 & 0.28 & 0.44 & 0.55 & 0.27 & 0.16 \\
\hline
\end{tabular}


Table.3 Grouping of rice genotypes based on seed length $(\mathrm{mm})$, seed width $(\mathrm{mm})$ and seed length / width ratio $(\mathrm{mm})$

\begin{tabular}{|c|c|c|c|}
\hline Sl.no. & Groups & $\begin{array}{l}\text { Number of } \\
\text { Genotypes }\end{array}$ & Name of the Genotype \\
\hline \multicolumn{4}{|c|}{ Seed length $(\mathrm{cm})$} \\
\hline 1. & Short $(6.1-8.5 \mathrm{~mm})$ & - & - \\
\hline 2. & Medium (8.6-10.5 mm) & 8 & $\begin{array}{l}\text { RYC-230, GNV-1405, GNV-1089, } \\
\text { GNV-05-01, GNV-1109, BPT-5204, } \\
\text { IET-18299 and IET-19251 }\end{array}$ \\
\hline 3. & Long (10.6-12.5 mm) & 9 & $\begin{array}{l}\text { GNV-1301, MT- } 4253, \text { IET-22066, } \\
\text { MT- 4021, MT- } 4420 \text {, MTU- 1010, } \\
\text { MT- 4541, MAS -26 and MAS-946- } \\
1\end{array}$ \\
\hline \multicolumn{4}{|c|}{ Seed width $(\mathrm{mm})$} \\
\hline 1. & Very narrow $<2.0 \mathrm{~mm}$ & - & - \\
\hline 2. & Narrow $(2.1-2.5 \mathrm{~mm})$ & - & - \\
\hline 3. & Medium (2.6-3.0 mm) & 6 & $\begin{array}{l}\text { RYC-230, GNV-1405, GNV-05-01, } \\
\text { BPT-5204, IET-18299 and IET- } \\
19251\end{array}$ \\
\hline 4 & Broad $(3.1-3.5 \mathrm{~mm})$ & 1 & GNV-1089 \\
\hline \multicolumn{4}{|c|}{ Seed length / width ratio (mm) } \\
\hline 1. & Semi spherical & 4 & $\begin{array}{l}\text { MT-4253, MT-4021, MT-4420 and } \\
\text { MTU-1010 }\end{array}$ \\
\hline 2. & Semi long & 5 & $\begin{array}{l}\text { GNV-1409, IET-22066, MT-4541, } \\
\text { MAS-26 and MAS-946-1 }\end{array}$ \\
\hline 3. & Elongated & 8 & $\begin{array}{l}\text { RYC-230, GNV-1405, GNV-1089, } \\
\text { GNV-1305, BPT-5204, IET-18299 } \\
\text { and IET-19251 }\end{array}$ \\
\hline
\end{tabular}

Table.4 Identification of rice genotypes through chemical tests

\begin{tabular}{|l|c|c|c|c|c|}
\hline \multicolumn{1}{|c|}{ Genotypes } & Phenol test & FeSO4 test & KOH test & NaOH test & Peroxidase test \\
\hline $\mathbf{G}_{\mathbf{1}}: \mathbf{R Y C - 2 3 0}$ & Brown & Grey Streaks & Light Yellow & Yellow & Absent \\
\hline $\mathbf{G}_{\mathbf{2}}: \mathbf{G N V - 1 4 0 5}$ & No colour & Grey Streaks & Light Yellow & Yellow & Absent \\
\hline $\mathbf{G}_{\mathbf{3}}: \mathbf{G N V - 1 0 8 9}$ & Dark Brown & No streaks & Light Yellow & Yellow & Absent \\
\hline $\mathbf{G}_{\mathbf{4}}: \mathbf{G N V - 1 3 0 1}$ & Brown & No streaks & Dark Brown & Yellow & Absent \\
\hline $\mathbf{G}_{\mathbf{5}}: \mathbf{G N V - 0 5 - 0 1}$ & Light Brown & No streaks & Light Yellow & Yellow & Absent \\
\hline $\mathbf{G}_{\mathbf{6}}: \mathbf{G N V - 1 1 0 9}$ & Brown & Brown Streaks & Light Yellow & Yellow & Absent \\
\hline $\mathbf{G}_{\mathbf{7}}: \mathbf{M T - 4 2 5 3}$ & Light Brown & Grey Streaks & Light Yellow & Yellow & Absent \\
\hline $\mathbf{G}_{\mathbf{8}}: \mathbf{I E T - 2 2 0 6 6}$ & Dark Brown & Brown Streaks & Light Yellow & Yellow & Absent \\
\hline $\mathbf{G}_{\mathbf{9}}: \mathbf{M T - 4 0 2 1}$ & Brown & Grey streaks & Light Yellow & Yellow & Absent \\
\hline $\mathbf{G}_{\mathbf{1 0}}: \mathbf{B P T - 5 2 0 4}$ & Light Brown & No streaks & Light brown & Yellow & Absent \\
\hline $\mathbf{G}_{\mathbf{1 1}}: \mathbf{I E T - 1 8 2 9 9}$ & No colour & Grey Streaks & Light brown & Yellow & Absent \\
\hline $\mathbf{G}_{\mathbf{1 2}}:$ MT-4420 & Light Brown & Brown Streaks & Light Yellow & Yellow & Absent \\
\hline $\mathbf{G}_{\mathbf{1 3}}:$ MTU-1010 & Light Brown & Brown Streaks & Dark Brown & Yellow & Absent \\
\hline $\mathbf{G}_{\mathbf{1 4}}:$ MT-4541 & No colour & No streaks & Light Yellow & Yellow & Absent \\
\hline $\mathbf{G}_{\mathbf{1 5}}: \mathbf{I E T - 1 9 2 5 1}$ & Light Brown & Grey Streaks & Light Yellow & Yellow & Absent \\
\hline $\mathbf{G}_{\mathbf{1 6}}:$ MAS-26 & Light Brown & No streaks & Light Yellow & Yellow & Absent \\
\hline $\mathbf{G}_{\mathbf{1 7}}:$ MAS-946-1 & Light Brown & Brown Streaks & Light Yellow & Yellow & Absent \\
\hline
\end{tabular}




\begin{tabular}{|l|}
\hline Phenol test: No colour, Light brown, Dark brown and Brown \\
\hline FeSO 4 test : Dark Grey streaks, Brown streaks and No streaks \\
\hline KaOH test: No colour, Light Yellow, Dark Brown, Brown \\
\hline NaOHtest : No Colour, Yellow Colour and Wine Red \\
\hline Peoxidasetest : Present, Absent \\
\hline
\end{tabular}

Table.5 DNA polymorphism generated using 22 SSR primers in 17 rice genotypes

\begin{tabular}{|c|c|c|c|c|c|c|}
\hline Sl. No. & SSR marker & $\begin{array}{c}\text { Expected } \\
\text { product size } \\
\text { (bp) }\end{array}$ & $\begin{array}{c}\text { Observed } \\
\text { product size } \\
\text { (bp) }\end{array}$ & $\begin{array}{c}\text { No. of a } \\
\text { Alleles } \\
\text { produced }\end{array}$ & $\begin{array}{l}\text { Major allele } \\
\text { frequency }\end{array}$ & $\begin{array}{c}\text { PIC } \\
\text { Value }\end{array}$ \\
\hline 1 & RM-241 & $100-170$ & $125-140$ & 3 & 0.70 & 0.46 \\
\hline 2 & RM-257 & $100-175$ & $140-150$ & 2 & 0.76 & 0.36 \\
\hline 3 & RM-224 & $150-180$ & $155-170$ & 3 & 0.53 & 0.61 \\
\hline 4 & RM-219 & $200-300$ & $190-210$ & 3 & 0.58 & 0.55 \\
\hline 5 & RM-252 & $215-254$ & $215-220$ & 3 & 0.52 & 0.58 \\
\hline 6 & RM-562 & 243 & $200-260$ & 3 & 0.58 & 0.55 \\
\hline 7 & RM-140 & 261 & $240-260$ & 3 & 0.41 & 0.65 \\
\hline 8 & RM-448 & 228 & $210-240$ & 3 & 0.41 & 0.66 \\
\hline 9 & RM-578 & $280-310$ & $300-320$ & 3 & 0.52 & 0.50 \\
\hline 10 & RM-286 & $98-122$ & $90-110$ & 3 & 0.52 & 0.55 \\
\hline 11 & OSR-28 & $120-140$ & $125-150$ & 3 & 0.64 & 0.52 \\
\hline 12 & RM-311 & 179 & $170-190$ & 2 & 0.76 & 0.36 \\
\hline 13 & RM-306 & 155 & $160-170$ & 2 & 0.58 & 0.48 \\
\hline 14 & RM-5389 & 132 & $120-130$ & 3 & 0.58 & 0.57 \\
\hline 15 & RM-161 & 187 & $180-190$ & 2 & 0.94 & 0.11 \\
\hline 16 & RM-44 & $100-130$ & $90-100$ & 3 & 0.52 & 0.55 \\
\hline 17 & RM-547 & 235 & $230-250$ & 3 & 0.82 & 0.30 \\
\hline 18 & RM-55 & $215-240$ & $200-220$ & 3 & 0.64 & 0.51 \\
\hline 19 & RM-231 & $168-185$ & $170-190$ & 3 & 0.58 & 0.57 \\
\hline 20 & RM-13 & $130-150$ & $130-150$ & 3 & 0.88 & 0.19 \\
\hline 21 & RM-452 & 105 & $105-110$ & 2 & 0.88 & 0.21 \\
\hline 22 & RM-171 & $320-345$ & $340-260$ & 3 & 0.52 & 0.60 \\
\hline \multicolumn{2}{|c|}{ Minimum } & 98 & 90 & 2 & 0.41 & 0.11 \\
\hline \multicolumn{2}{|c|}{ Maximum } & 345 & 260 & 3 & 0.88 & 0.66 \\
\hline \multicolumn{2}{|c|}{ Mean } & - & - & 2.5 & 0.6 & 0.5 \\
\hline
\end{tabular}



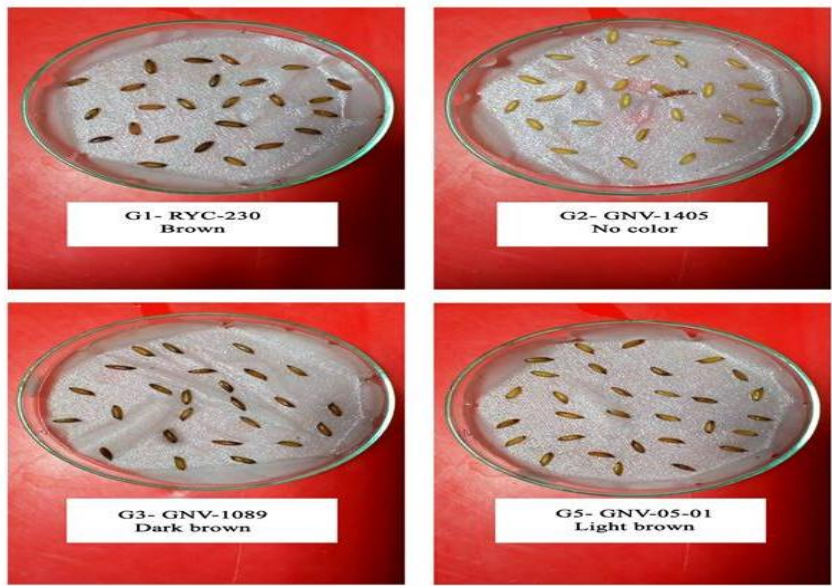

Fig. 1 Response of rice genotypes to Phenol test
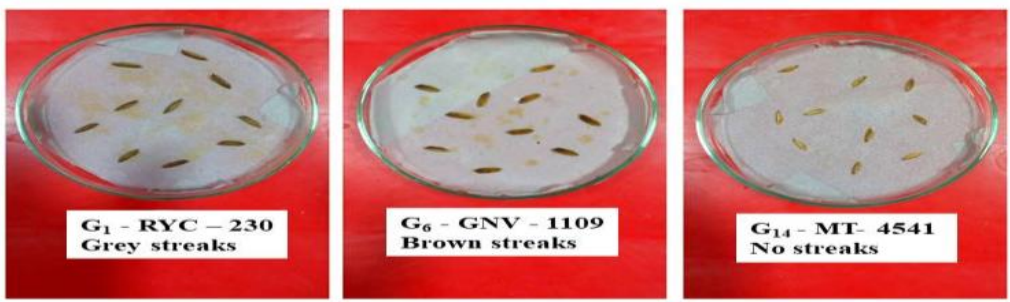

Fig.2 Response of rice genotypes to $\mathrm{FeSO}_{4}$ test

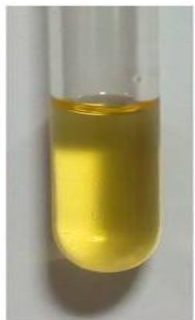

Gi1- RYC 230

ight yellow

KOH test

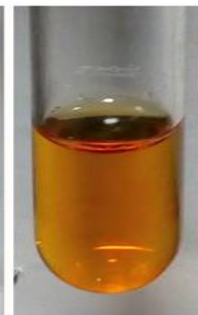

G10- BPT-52:04 Light brow

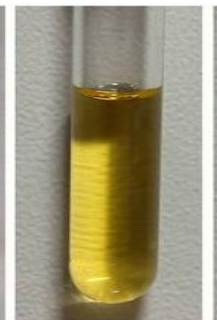

G3- GNV-1089

Yellow

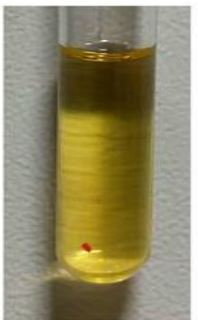

G10- BPT-5204

Fig. 3 Response of rice genotypes to $\mathrm{KOH}$ test and $\mathrm{NaOH}$ test
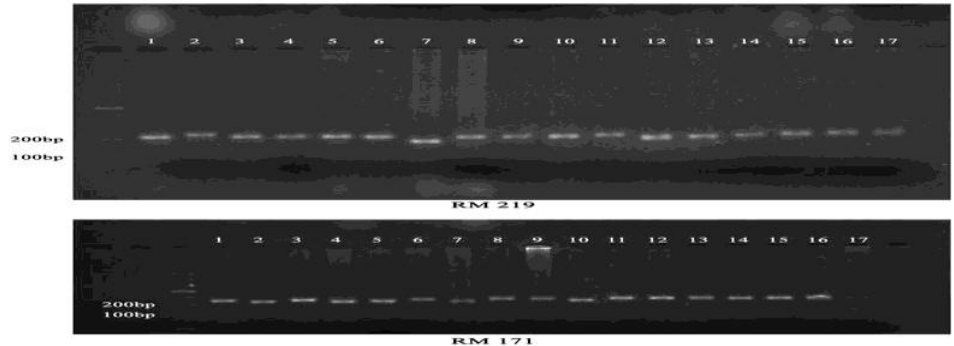

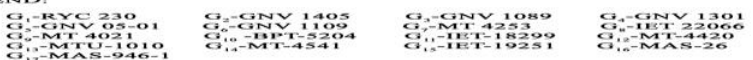

Fig. 4 SSR marker profile of rice genotypes generated by the primer RM 219 and RM 171 


\section{Ferrous sulphate $\left(\mathrm{FeSO}_{4}\right)$ test}

In ferrous sulphate test, the genotypes were grouped into two groups (No streaks, Grey streaks and Brown streaks). Six genotypes $\left(\mathrm{G}_{1}:\right.$ RYC-230, G $:$ GNV-1405, G 7 :MT - 4253, $\mathrm{G}_{9}: \mathrm{MT}-4021, \mathrm{G}_{11}: \mathrm{IET}-18299$ and $\mathrm{G}_{15}:$ IET19251) showed grey streaks, six genotypes $\left(\mathrm{G}_{3}: \mathrm{GNV}-1089, \mathrm{G}_{4}: \mathrm{GNV}-1301, \mathrm{G}_{5}: \mathrm{GNV}-05-\right.$ $01, \quad \mathrm{G}_{10}$ :BPT-5204, $\mathrm{G}_{14}$ :MT-4541 and $\mathrm{G}_{16}$ :MAS-26) showed no streaks and five genotypes $\left(\mathrm{G}_{6}: \mathrm{GNV}-1109, \mathrm{G}_{8}:\right.$ IET 22066, $\mathrm{G}_{12}$ :MT-4420, $\mathrm{G}_{13}$ :MTU-1010 and $\mathrm{G}_{17}$ :MAS946-1) (Fig. 2) showed brown streaks. The similar grouping was also reported by Gupta and Agrawal (1988) in paddy, Saharan (1991) in paddy, Ponnusamy et al., (2003) in cotton and Mathad et al., (2019) in pigeon pea using pixel luminance test.

\section{Potassium hydroxide (KOH) test}

In the case of potassium hydroxide test, the genotypes showed a positive response to this test, as well they turned yellow colour solution. However, the intensity of colour varied among the varieties. Out of seventeen genotypes, two genotypes ( $\mathrm{G}_{4}$ : GNV-1301 and $\mathrm{G}_{13}$ : MTU-1010) (Fig. 3) have shown dark brown colour change and the rest of the genotypes reacted to light yellow colour. Similar groupings were reported by Mckee (1973) in wheat, Rosta (1975), SambasivaRao et al (2002) in groundnut and BiradarPatil et al., (2006) in safflower genotypes and Masuthi et al., (2015) in rice. The varied colour reaction may be due to the chemical composition of seed or selective action of enzymes present which may be governed genetically.

\section{Sodium hydroxide $(\mathrm{NaOH})$ test}

All the 17 rice genotypes showed yellow colour reaction with sodium hydroxide solution (Fig. 3). Similar results were reported by Sambasivarao et al., (2002) in rice, Ponnuswamy et al., (2003) in cotton and BiradarPatil (2006) in safflower genotypes, Tiwari et al., (2013) in rice, Masuthi et al., (2015) in rice and Mathad et al., (2019) in pigeon pea. The colour reaction to sodium hydroxide solution was obtained due to the reaction of seeds to secondary metabolites (Vanderburg and Vanzwol, 1991). The difference in colour reaction of seeds seems to be due to differences in the genetic background concerning the enzyme system (Chakrabarthy and Agrawal, 1990). Potassium hydroxide and sodium hydroxide tests are useful to distinguish red seed varieties from white seed varieties if the seed coat colour of red seeded varieties vanished due to unfavourable climate condition. Vanagamudi et al., (1988) reported that among 85 rice varieties, 71 varieties showed negative reaction to these chemicals and remaining varieties showed deep-wine red staining.

\section{Peroxidase $\left(\mathrm{H}_{2} \mathrm{O}_{2}\right)$ test}

All the genotypes studied showed the negative reaction to peroxidase activity. Similar negativity of the reaction was also reported by Hiremath (2013) in rice genotypes. The results of peroxidase test are not conformity with previous work reported by Chakrabarthy and Agrawal (1989) in black gram, Agarwal and Pawar (1990) in soybean and Kirankumar Reddy (2004) in cotton,

\section{Identification of rice genotypes using SSR molecular markers}

In the present study, 22 SSR primers were used which produced scorable, unambiguous markers. A total of 61 alleles were produced and all are polymorphic(Fig. 4). The number of alleles detected per primer pair ranged from 2 in five primers to 3 in rest of 17 primers with an average of 2.77 . The SSR 
products size ranged from 90 to $340 \mathrm{bp}$. The alleles showed a high degree of polymorphism. PIC value varied significantly for all the studied SSR loci. In the present study, the level of polymorphism among the 17 genotypes was evaluated by calculating PIC values for each of the SSR loci. The PIC value ranged from 0.11 (RM161) to 0.66 (RM 448 ) with an average of 0.46 per marker.

Markers with PIC values of 0.5 or higher are highly informative for genetic studies and are extremely useful in distinguishing the polymorphism rate of a marker at a specific locus. This indicated that the genotypes used in the present study were more diverse due to differences in their genetic constitution. Presence of polymorphism between genotypes revealed that the presence of genetic diversity at the molecular level was high among the selected genotype which implies that, the genetic diversity is more than the morphological diversity and there is ample scope to utilize the material in the breeding programme. These results corroborate the earlier findings of Shahriar et al., (2014), Kundur et al., (2015) and Maruthi (2016) in rice.

\section{Acknowledgements}

This research was sponsored by UGC-NFSC Scheme, Government of India.

\section{References}

Abrol, Y. P. and Uprety, D. C., 1972.Phenol colour reaction for discolouration of paddy. Bull. Grain Tech, 10: 288-290.

Agarwal, R. L. and Pawar, A., 1990. Identification of soybean varieties based on seed and seedling characteristics. Seed Research, 18 (1): 77-81.

Anitalakshmi, V., Gowda, R., Sathisha, C. S. and Rajendra, P., 2014. Varietal response to various chemical tests for their characterization in rice. Indian Journal of Plant Sciences, 3: 2319-3824. Anuradha, R., Balamurugan, P., Srimathi, P. and Sumathi, S., 2009. Influence of seed size on seed quality of chickpea (Cicerarietinum L.). Legume Res. Int. J., 32:133-135.

Ashwani, K., Chowdhary, R. K., Kapoor, R. L. and Dahiya, O. S., 1995.Identification of pearl millet hybrids and their parental lines using seeds and seedling characters, chemical tests and gel electrophoresis. Seed Science and Technology., 23: 21-32.

BiradarPatil, N. K., SangeetaMacha, Motagi, B. N., Vijaykumar, A. G. and Hanchinal, R. R., 2006, Characterization of safflower varieties through chemical tests. Abst.XII Nation. Seed Sem., 24-26 February, ANGRAU, Hyderabad, p.168.

Bora, R., Deka, S. D. and Sen, P., 2008. Identification of rice varieties of Assam based on grain characters and reaction to certain chemical tests. Seed Research, 36: 51-55.

Buttery, B. R. and Buzzell, R. I., 1968, Peroxidase activity in seeds of soybean varieties. Crop Sci., 8: 722-724.

Cao, D. and Oard, J. H., 1997. Pedigree and RAPD-based DNA analysis of commercial U.S. Rice cultivars. Crop Sci., 37: 1630-1635.

Chakrabarthy, S. K. and Agarwal, R. L., 1990. Identification of blackgram varieties- III: Utilization of seedling growth response to added chemical. Seed Res., 18 (1): 24-31.

Chauhan, J. S. and Nanda, J. S., 1983, Genetic variability for physic-chemical characters of rice grain in segregation $O$. Sativa L. Oryza. 20:209-215.

Devi Singh, Anuj Kumar, Navneet Kumar, Vipin Kumar, Sachin Kumar, AmalBharadwaj, PreetiSirohi and Chand, S., 2011. Comparison of traditional physic-chemical methods and 
molecular marker assays for characterization of Basmati rice (Oryzasativa L.). African J. of Biotechnology.10(62):13390-13398.

Gupta, P. K. and Agarwal, R. L., 1988. Determination of varietal purity of paddy varieties by laboratory evaluation. Oryza. 25: 310-314.

Hiremath, M., 2013. Characterization of paddy genotypes through image analyzer. M. Sc., (Ag.) theses, UAS Raichur, Karnataka.

Iwata, H., Ebana, K., Uga, Y., Hayashi, T. and Jannink, J. L., 2010.Genomic-wide association study of grain shape among Oryzasativa L. germplasms based on elliptic Fourier analysis. Mol. Breed., 25:2013-215.

Jaiswal, J. P. and Agarwal, R. L., 1995. Varietal purity determinations in rice modification of the phenol test. Seed Sci. \& Technol., 23: 33-42.

Joshi, M. G. and Banerjee, S. K., 1970. Genetics of phenol colour reaction in emmer wheat. Association of Official Seed Analyst, p. 28. Journal of Applied and Natural Science.6 (1): 258-261.

Kirankumar Reddy, C., 2004.Studies on laboratory techniques for identification of cotton (Gossypium spp.) genotypes. M.Sc. (Agri.) Thesis, Acharya N.G. Ranga Agric. Univ., Hyderabad.

Kundur, P. J., Prakash, G., Patil, B. G., Harish, C. K., Ramesh and. Shahidhar, H. E., 2015.Molecular characterization of rice (Oryzasativa L.) genotypes using target region amplification polymorphism (TRAP) markers in relation to grain iron content.IJAAR. 7(1):125-133.

Maruthi, J. B., 2016. Evaluation and enrichment of seed micronutrient (Fe and $\mathrm{Zn}$ ) content through biofortification in paddy genotypes. Ph. D (Ag.) theses, UAS Raichur, Karnataka.

Masuthi, D. A., Vyakarnahal, B. S.,
Deshpande, V. K., Jagadeesha, R. C., Mukesh, L. C. and Khaja, R. S., 2015.Characterization of traditional aromatic rice cultivars by chemical markers. Int. J. of Advanced Res., 3: 103-107.

Mathad, R. C., Channaveeraswamy, A. S., Vinod Kumar, D., RudraNaik, V. and Ashtaputre, S. A., 2019. Faster Chemical Methods to Determine Genetic Purity in Pigeon Pea (Cajanuscajan L. Mill sp.). Int.J.Curr.Microbiol.App.Sci., $\quad$ 8(3): 1378-1392.

McKee, 1973. Chemical and biochemical techniques for varietal identification. Seed Sci. Technol., 1: 181-199.

Meshram, L. D. and Rahangdale, S. L., 1988, Studies on phenol test in rice. Ann. Pl. Physiol., 2 (1):107-109.

Paulsen, M. R., Wigger, W. D., Litchfield, J. B. and Sinclair, J. B., 1989.Computer image analysis for detection of maize and soybean kernel quality factors. J. Agric. Eng. Res., 43: 93-101.

Ponnuswamy, A. S., Bhasakaran, M. and Sashri, G., 2003. Variety characterization in cotton by physical, chemical and biochemical methods. Training Manual on Varietal Characterization by Image Analysis and Electrophoresis.106-120.

Raju, P., Patil, S. B., Vasudevan, S. N., Ibrahim, Md. and Mathad, R. C., 2017. Characterization of parents and $F_{1}$ seeds of rice hybrid $\mathrm{KRH}-4$ using different chemical tests and seed image analyzer. Agric. Update., 12 (TECHSEAR-5), 1261-1265.

Rosta, K., 1975. Variety determination in rice. Seed Science and Technology., 3: 161 168.

Saharan, V., 1991. Varietal identification in rice. M. Sc. (Agri.) Thesis, Haryana Agric. Univ., Hissar (India).

Sainis, J. K., Shouche, S. P. and Bhagat, S. G., 2009. Image analysis of wheat grains 
developed in different environment and its implications for identification. J. Agric. Sci., 144(3): 221-227.

SambasivaRao, P., Murali Mohan Reddy, B., Bharathi, M. and Bayyapu Reddy, K., 2002, Varietal identification in groundnut (Arachishypogaea L.) by chemical tests and electrophoresis of total soluble seed proteins. Seed Tech. News., 32(1): 93.

Shahriar, M. H., Robin, A. H. K., Begum, S. $\mathrm{N}$ and Hoque, A., 2014. Diversity analysis of some selected rice genotypes through SSR based molecular markers. J. Bangladesh Agril. Univ., 12(2): $307-$ 311.

Suchowilska, E. and Wiwari, M., 2006. Multivariate analysis of image descriptors of common wheat (Triticumaestivum) and spelt (Triticumspelta) grain infected by Fusariumculmorum. Int. Agrophysics. 20: 345-351.

Takahasi, N. and Hamaz, A. H. A., 1983.Diffrentiation of ecotypes in Oryzasativa L. I: Re-examination for colour reaction with phenol. Japan J. Breed., 33(3):243-250.

Tiwari, J. K., Rastogi, N. K., Chandrakar, P. K., Sarawgi, A. K. and Verulkar, S. B., 2013.Identification of rice varieties through chemical tests. Seed Res., 41(1):83-90.

Utku, H., Koksel, H. and Ozakara, R., 1998.
Classification of barleys based on malting quality by image analysis. J. Inst. Brew., 104: 351-354.

Vanagamudi, K., Palaniswamy, V. and Natesan, 1988. Variety identification in rice: Examination of hulled grain. Seed Sci. Technol., 16 (2): 465-470.

Vanderburg, N. J. and Vanzwol, R. A., 1991. Rapid identification techniques used in laboratories of the International Seed Testing Association: a survey. Seed Sci Technol., 19: 687-700.

Vijayalakshmi, B. and Vijay, D., 2009. Development of Seed Keys for Varietal Identification in Rice (Oryzasativa) using Chemical Tests.Seed Research. 37 (1\&2): 56-61.

Walls, W. E., 1965.A standardized phenol method for testing wheat seed for varietal purity.

Wang, J. and Shen, Z., 1992. Study on the classification of American rice varieties. Crop Gen. Resour., 2:4-6.

Zayas, I. Y., Martin, C. R., Steele, J. L. and Katsevich, A., 1996. Wheat classification using image analysis and crush force parameters. Ameri.Soci. Agric. Eng., 39 (6): 2199-2204.

Zayas, I. Y., Pomeranz, Y. and Lai, F. S., 1989.Discrimination of wheat and non wheat components in grain sample by using image analysis. Cereal Chem., 66 (3): 233-237.

\section{How to cite this article:}

Sudeep Kumar, E., S. N. Vasudevan, N. M. Shakuntala, S. R. Doddagoudar, B. G. MasthanReddy and Mahantashivayogayya, K. 2021. Characterization of Rice Genotypes Based on Physical, Chemical and Molecular Methods. Int.J.Curr.Microbiol.App.Sci. 10(01): 14801492. doi: https://doi.org/10.20546/ijcmas.2021.1001.176 